\title{
An Explicit Addition Formula for the Inverse Jacobian Elliptic Functions
}

\author{
By Henry E. Fettis
}

\begin{abstract}
An explicit addition formula is derived for the inverse sine-amplitude function. This is used to obtain an explicit formula for the inverse sine-amplitude function with complex argument.
\end{abstract}

Although addition formulae for the Jacobian elliptic functions $\operatorname{am}(u) \operatorname{sn}(u)$, $\operatorname{cn}(u) \operatorname{dn}(u)$ are given in most standard texts, e.g. [1], similar expressions for the inverse functions seem not to be well known. In the present note, it is shown that an explicit formula for $\operatorname{sn}^{-1}(\alpha+\beta)$ can be found from the known addition formula for the amplitude function.

By definition, if*

$$
\operatorname{sn}^{-1}(\alpha+\beta)=u+v,
$$

then

$$
\alpha+\beta=\sin (x+y)
$$

where

$$
x+y=\operatorname{am}(u+v) .
$$

Using the addition formula for $\operatorname{am}(u+v)$ [1, formula 123.01], we find

$$
\tan x=\operatorname{tn} u \operatorname{dn}(v), \quad \tan y=\operatorname{tn} v \operatorname{dn}(u) .
$$

The system (4) is easily solved by eliminating either $\operatorname{sn}(v)$ or $\operatorname{sn}(u)$. The resultants are biquadratics for $\operatorname{sn}(u)$ or $\operatorname{sn}(v)$, e.g.,

$$
\begin{aligned}
& k^{2} \cos ^{2} y \operatorname{sn}^{4} u-\left[1+k^{2}\left(\sin ^{2} x \cos ^{2} y-\cos ^{2} x \sin ^{2} y\right)\right] \operatorname{sn}^{2} u+\sin ^{2} x=0, \\
& k^{2} \cos ^{2} x \operatorname{sn}^{4} v-\left[1+k^{2}\left(\cos ^{2} x \sin ^{2} y-\sin ^{2} x \cos ^{2} y\right)\right] \operatorname{sn}^{2} v+\sin ^{2} y=0 .
\end{aligned}
$$

Solving gives**

$$
\operatorname{sn}(u)=\frac{\left((1+k \sin x \cos y)^{2}-k^{2} \cos ^{2} x \sin ^{2} y\right)^{1 / 2}}{2 k \cos y}
$$

$$
-\frac{\left((1-k \sin x \cos y)^{2}-k^{2} \cos ^{2} x \sin ^{2} y\right)^{1 / 2}}{2 k \cos y}
$$

Received February 10, 1972.

AMS 1970 subject classifications. Primary 33A25.

* The modulus is assumed to be " $k$ " unless otherwise noted.

** The negative sign is chosen in order to obtain a positive solution which remains finite as $k \rightarrow 0$.

Copyright (c) 1972, American Mathematical Society 


$$
\begin{gathered}
\operatorname{sn}(v)=\frac{\left((1+k \cos x \sin y)^{2}-k^{2} \sin ^{2} x \cos ^{2} y\right)^{1 / 2}}{2 k \cos x} \\
\quad-\frac{\left((1-k \cos x \sin y)^{2}-k^{2} \sin ^{2} y \cos ^{2} y\right)^{1 / 2}}{2 k \cos x} .
\end{gathered}
$$

Further, from Eq. (2),

$$
\begin{gathered}
\cos x=\frac{1}{2}\left[\left((1+\beta)^{2}-\alpha^{2}\right)^{1 / 2}+\left((1-\beta)^{2}-\alpha^{2}\right)^{1 / 2}\right] \\
\cos y=\frac{1}{2}\left[\left((1+\alpha)^{2}-\beta^{2}\right)^{1 / 2}+\left((1-\alpha)^{2}-\beta^{2}\right)^{1 / 2}\right] \\
\sin x \cos y=\alpha \\
\cos x \sin y=\beta .
\end{gathered}
$$

Hence, finally

$$
\begin{aligned}
\operatorname{sn}^{-1}(\alpha \pm \beta)= & \operatorname{sn}^{-1}\left\{\frac{\left((1+k \alpha)^{2}-k^{2} \beta^{2}\right)^{1 / 2}-\left((1-k \alpha)^{2}-k^{2} \beta^{2}\right)^{1 / 2}}{k\left[\left((1+\alpha)^{2}-\beta^{2}\right)^{1 / 2}+\left((1-\alpha)^{2}-\beta^{2}\right)^{1 / 2}\right]}\right\} \\
& \pm \operatorname{sn}^{-1}\left\{\frac{\left((1+k \beta)^{2}-k^{2} \alpha^{2}\right)^{1 / 2}-\left((1-k \beta)^{2}-k^{2} \alpha^{2}\right)^{1 / 2}}{k\left[\left((1+\beta)^{2}-\alpha^{2}\right)^{1 / 2}+\left((1-\beta)^{2}-\alpha^{2}\right)^{1 / 2}\right]}\right\} \\
= & \operatorname{sn}^{-1}\left\{\frac{\left((1+\alpha)^{2}-\beta^{2}\right)^{1 / 2}-\left((1-\alpha)^{2}-\beta^{2}\right)^{1 / 2}}{\left((1+k \alpha)^{2}-k^{2} \beta^{2}\right)^{1 / 2}+\left((1-k \alpha)^{2}-k^{2} \beta^{2}\right)^{1 / 2}}\right\} \\
& \pm \operatorname{sn}^{-1}\left\{\frac{\left((1+\beta)^{2}-\alpha^{2}\right)^{1 / 2}-\left((1-\beta)^{2}-\alpha^{2}\right)^{1 / 2}}{\left((1+k \beta)^{2}-k^{2} \alpha^{2}\right)^{1 / 2}+\left((1-k \beta)^{2}-k^{2} \alpha^{2}\right)^{1 / 2}}\right\}
\end{aligned}
$$

The above formulae provide a means of explicit calculation of the inverse Jacobian functions in the complex plane by replacing $\beta$ by $i \beta, v$ by $i v$, and using Jacobi's imaginary transformation. The real part involves only real quantities, whereas the imaginary part involves complex numbers under the radicals. It is therefore simpler to calculate the imaginary part from the equivalent of Eq. (4). Specifically, we find

$$
\mathrm{sn}^{-1}(\alpha+i \beta)=u+i v,
$$

where

$$
\begin{aligned}
\operatorname{sn}(u, k) & =\frac{\alpha}{\cosh y\left\{\left((1+k \alpha)^{2}+(k \beta)^{2}\right)^{1 / 2}+\left((1-k \alpha)^{2}+(k \beta)^{2}\right)^{1 / 2}\right\}}, \\
\operatorname{sn}\left(v, k^{\prime}\right) & =\frac{\tanh y}{\left(1-k^{2} \operatorname{sn}^{2} u\right)^{1 / 2}}
\end{aligned}
$$

and

$$
\cosh y=\frac{1}{2}\left[\left((1+\alpha)^{2}+\beta^{2}\right)^{1 / 2}+\left((1-\alpha)^{2}+\beta^{2}\right)^{1 / 2}\right] .
$$

The case where $\beta=0$ is of special interest, since Eq. (10) simplifies to

$$
\begin{gathered}
\operatorname{sn}(u, k)=\frac{(1+k \alpha)-|1-k \alpha|}{2 k \cosh y}, \quad \operatorname{sn}\left(v, k^{\prime}\right)=\frac{\tanh y}{\left(1-k^{2} \operatorname{sn}^{2} u\right)^{1 / 2}}, \\
\cosh y=\frac{1}{2}[(1+\alpha)+|1-\alpha|] .
\end{gathered}
$$


If $\alpha<1$, the above gives

$$
\cosh y=1, \quad \operatorname{sn}(u, k)=\alpha, \quad \operatorname{sn}\left(v, k^{\prime}\right)=0 .
$$

Hence, $u=F(\alpha, k), v=0$.

If $\alpha>1$ and $k \alpha>1$, we find

$$
\begin{gathered}
\cosh y=\alpha, \quad \tanh y=\left(\alpha^{2}-1\right)^{1 / 2} / \alpha, \\
\operatorname{sn}(u, k)=1 / k \alpha, \quad \operatorname{sn}\left(v, k^{\prime}\right)=1,
\end{gathered}
$$

whence

$$
u+i v=F\left[\sin ^{-1}(1 / k \alpha), k\right]+i K^{\prime}(k) .
$$

Finally, if $\alpha>1$ and $k \alpha<1$, Eqs. (12) and (13) give

$$
\cosh y=\alpha, \quad \operatorname{sn}(u, k)=1, \quad \operatorname{sn}\left(v, k^{\prime}\right)=\left(\alpha^{2}-1\right)^{1 / 2} / \alpha k^{\prime}
$$

and

$$
u+i v=K+i F\left[\sin ^{-1}\left(\left(\alpha^{2}-1\right)^{1 / 2} / \alpha k^{\prime}\right), k^{\prime}\right]
$$

Aerospace Research Laboratories

Air Force Systems Command

United States Air Force

Wright-Patterson Air Force Base, Ohio

1. P. F. BYRD \& M. D. FRIEDMAN, Handbook of Elliptic Integrals for Engineers and Scientists, 2nd rev. ed., Die Grundlehren der math. Wissenschaften, Band 67, Springer-Verlag, New York, 1971. MR 43 \#3506. 